\title{
openheart Contribution of the long-term care insurance certificate for predicting 1-year all-cause readmission compared with validated risk scores in elderly patients with heart failure
}

\author{
Kayo Takahashi, ${ }^{1,2}$ Makoto Saito, ${ }^{1}$ Shinji Inaba, ${ }^{1}$ Toru Morofuji, ${ }^{1}$ Hiroe Aisu, ${ }^{1,2}$
} Takumi Sumimoto, ${ }^{1}$ Akiyoshi Ogimoto, ${ }^{3}$ Shuntaro Ikeda, ${ }^{2}$ Jitsuo Higaki ${ }^{2}$

To cite: Takahashi $\mathrm{K}$, Saito M, Inaba S, et al. Contribution of the long-term care insurance certificate for predicting 1-year all-cause readmission compared with validated risk scores in elderly patients with heart failure. Open Heart 2016;3: e000501. doi:10.1136/ openhrt-2016-000501

- Additional material is available. To view please visit the journal (http://dx.doi.org/ 10.1136/openhrt-2016000501)

Received 15 July 2016 Revised 19 October 2016 Accepted 1 November 2016

\section{(a) CrossMark}

${ }^{1}$ Kitaishikai Hospital, Ozu, Japan

${ }^{2}$ Ehime University Graduate School of Medicine, Toon, Japan

${ }^{3}$ Uwajima City Hospital, Uwajima, Japan

Correspondence to Dr Makoto Saito; saito0321jp@yahoo.co.jp

\section{ABSTRACT}

Objectives: Readmission is a common and serious problem associated with heart failure (HF).

Unfortunately, conventional risk models have limited predictive value for predicting readmission. The recipients of long-term care insurance (LTCI) are frail and have mental and physical impairments. We hypothesised that adjustment of the conventional risk score with an LTCI certificate enables a more accurate appreciation of readmission for HF.

Methods: We investigated 452 patients with HF who were followed up for 1 year to determine all-cause readmission. We obtained their clinical and socioeconomic data, including LTCI. The three clinical risk scores used in our evaluation were Keenan (2008), Krumholz (2000) and Charlson (1994). We used net reclassification improvement (NRI) to assess the incremental benefit.

Results: Patients with LTCI were significantly older, and had a higher prevalence of cerebrovascular disease and dementia than those without LTCI. One-year allcause readmission ( $n=193,43 \%$ ) was significantly associated with all risk scores, receiving $\mathrm{LTCl}$ and the category of LTCI. Receiving LTCI was associated with readmission independent of all risk scores (HR, 1.59 to 1.63; all $p<0.01$ ). Adding $L T C I$ to all risk scores led to a significantly improved reclassification, which was observed in the subgroup of patients with HF with preserved ejection fraction $(\geq 50 \%)$ but not in the subgroup with reduced ejection fraction $(<50 \%)$.

Conclusions: Possession of an LTCI certificate was independently associated with 1-year all-cause readmission after adjusting for validated clinical risk scores in patients with HF. Adding LTCI status significantly improved the model performance for readmission risk, particularly in patients with $\mathrm{HF}$ and preserved ejection fraction.

\section{INTRODUCTION}

Readmission of patients with heart failure (HF) following hospital discharge is frequent

\section{KEY QUESTIONS}

What is already known about this subject?

- Heart failure (HF) readmissions are a common and serious problem of heterogeneous aetiology.

- Conventional risk models have limited predictive value for predicting readmission for HF.

- The recipients of long-term care insurance (LTCI) are frail and have mental and physical impairments.

- Frailty predicts adverse outcomes in several settings.

What does this study add?

- An LTCI certificate was associated with 1-year all-cause readmission, independent of conventional clinical risk scores for HF.

- An LTCI certificate provides incremental value over validated clinical risk scores for predicting readmission for $\mathrm{HF}$.

- This incremental benefit was observed particularly in patients with HF with preserved ejection fraction.

How might this impact on clinical practice?

- Risk stratification using the adjusted clinical risk score with an LTCI certificate might enable targeting of resources to prevent middle-term readmission in the highest risk HF.

and represents a major problem for achieving a successful treatment outcome. ${ }^{1}{ }^{2}$ Moreover, readmission is a powerful independent risk factor for death among patients with $\mathrm{HF}$ and a serious medical economic problem. ${ }^{1}$ Risk stratification for readmission of patients with HF might enable targeting of resources to prevent readmission of patients at the highest risk. Several validated risk models and scores are available to predict readmission of patients with $\mathrm{HF}^{3-7}$ Unfortunately, these models, which mainly 
comprise clinical parameters and comorbidities, have limited predictive value because the problem of readmission is a complicated sociobiological process. ${ }^{8}$

Recently, socioeconomic parameters have emerged as contributors to predicting readmission of patients with $\mathrm{HF}^{8-10}$ In response to the ageing society, long-term care insurance (LTCI) has been successively introduced to provide social care for the frail and elderly to lead more independent lives in several countries including Japan, Germany, South Korea, the Netherlands and the Nordic countries. ${ }^{11-13}$ Thanks to this LTCI, the recipient can take advantage of home-based and institutional care at a relatively affordable cost. Therefore, the eligibility criteria for LTCI is strictly managed using questionnaires and interviews conducted by experts such as physicians, physiotherapists, social workers, health welfare professionals and administrators who assess an applicant's frailty and dementia. Conversely, a certificate in LTCI indicates that the recipients are demonstrably frail and have mental and physical impairments requiring sufficient social support. Frailty predicts adverse outcomes in several settings. ${ }^{311}{ }^{14-19}$ Thus, an LTCI certificate might be associated with outcomes beyond providing social benefit. Therefore, we investigated whether the adjusted conventional clinical risk scores plus an LTCI certificate would enable a more accurate appreciation of 1-year unplanned all-cause readmission for HF.

\section{METHODS}

\section{Study design}

To enrol the patients, we used Kitaishikai Hospital's admission database, which was originally created by hospital staff and includes all admitted patients and their primary diagnosis code International Classification of Diseases, 10th Revision (ICD-10) related to the index admission. Using this database, we retrospectively identified 525 consecutive adult patients (age range, 3090 years) who were admitted to Kitaishikai Hospital in Ozu City from July 2006 to June 2014 with a primary diagnosis of congestive HF (ICD-10 codes, I500 (Congestive heart failure) I501 (Left ventricular failure) and I509 (Heart failure, unspecified)). Ozu City is located in a rural area of Japan and its adult population is small (around 35000 people). Kitaishikai Hospital is a core hospital in Ozu City that supports physicians, and most regular patients are admitted to Kitaishikai Hospital. Further, only Kitaishikai Hospital offers cardiology services in this medical district. The value of these data for retrospective study depends on the accuracy of input, ${ }^{20}$ so validation is critical. Two cardiologists (KH and $\mathrm{TM}$ ) independently reviewed each patient's medical records to confirm the diagnosis of HF. When agreement was not obtained, a third cardiologist (TS) decided on the issue and an agreement was obtained. HF was defined by a combination of typical signs or symptoms of HF, such as breathlessness at rest or during exertion, ankle oedema, pulmonary crepitations and objective evidence of cardiac dysfunction (chest X-ray, echocardiography). ${ }^{21}$ Patients admitted for main reasons other than HF were excluded $(n=73)$. The most common reason for exclusion was coronary artery disease $(n=29)$, followed by hospital transfer at the date of admission $(n=9)$, renal failure $(n=8)$, workup $(n=5)$, depression $(n=4)$, arrhythmia $(n=4)$, dehydration $(n=4)$, lung disease $(n=4)$, cancer $(n=3)$ and cerebrovascular disease $(n=3)$. Data for 452 patients were included in the study, which was approved by the Ethics Committee of Ehime University Graduate School of Medicine.

\section{Clinical data}

Using each patient's medical record, we assessed sociodemographic variables, comorbidities, medical history, medication at discharge, vital signs at discharge, serum markers and echocardiographic data acquired closest to discharge.

\section{Risk scores}

We calculated three conventional risk scores to predict readmission of each patient with $\mathrm{HF}$ as follows: score 1 , Keenan et $a l^{5}{ }^{5}$ score 2, Krumholz et $a l ;^{6}$ and score 3, Charlson et $a .^{22}$ Although Score 1 was originally designed as a measure of the risk of 30-day all-cause readmission or death, it was measured because the score comprehensively included the long-term clinical risks for readmission for $\mathrm{HF}$ and validated in huge cohort. $^{5} 2324$ In addition, Score 3 was originally designed as a measure of the risk of 1-year mortality attributed to comorbidity as determined by a longitudinal study, but it was measured because it is widely used by health researchers to measure burden of disease and case mix.

Score 1 (The Yale Center for Outcome Research and Evaluation (CORE) Readmission Risk Score for HF, referred to as the Yale score) indicates the probability of readmission and was calculated using the intercept and regression coefficient of the 19 following variables: (1) male; (2) age; (3) chronic obstructive pulmonary disease; (4) dementia/Alzheimer's disease; (5) diabetes; (6) cerebrovascular event; (7) congestive heart failure; (8) history of percutaneous transluminal coronary angioplasty; (9) history of coronary artery disease; (10) systolic blood pressure; (11) respiratory rate; (12) heart rate; (13) cardiac arrest; (14) EF; (15) aortic stenosis ( $\geq$ moderate); (16) sodium; (17) blood urea nitrogen and creatinine; (18) glucose; and (19) haematocrit (http:// www.readmissionscore.org/heart_failure.php) ${ }^{5}$ First, we calculated the logit for each patient by summing the intercept and each regression coefficient. Then we acquired the probability (Yale score) by converting the logit. Since the Yale score is intended for use for patients aged $\geq 65$ years, we assigned 0 to the regression coefficient of the variable (2) 'Age' for patients aged $<65$ years.

Score 2 (0 and 4 indicating the lowest and highest, respectively) assigned points according to four variables. 
One point was added to each of the variables as follows: hospitalisation during the preceding year; history of $\mathrm{HF}$ or diabetes mellitus; and serum creatinine $>2.5 \mathrm{mg} / \mathrm{dL}$ on discharge. In the present study, the history of prior HF admission before the index admission was confirmed through chart review, and this was used as a 'history of HF'.

Score 3 (The Charlson Comorbidity Index, with 0 and 37 indicating the lowest and highest, respectively) assigns points according to the 19 variables listed in Score 1. One point was added for each of the 10 variables as follows: (1) myocardial infarction, (2) congestive heart failure, (3) peripheral vascular disease, (4) cerebrovascular disease, (5) dementia, (6) chronic pulmonary disease, (7) connective tissue disease, (8) ulcer, (9) mild liver disease and (10) diabetes. Two points were added for each of the six following variables: (1) hemiplegia, (2) moderate or severe renal disease, (3) diabetes with end organ damage, (4) any tumour, (5) leukaemia and (6) lymphoma. Three points were added for (1) moderate or severe liver disease, while six points were added for (1) metastatic solid tumour and (2) AIDS.

\section{Echocardiography}

All patients were examined with echocardiography and most echocardiographic data were available (missing in $<5 \%$ of patients). Transthoracic echocardiography was performed using Vivid 7 or Vivid E9 Ultrasound Systems (GE Vingmed, Horten, Norway). Echocardiographic images were digitally recorded and downloaded to an imaging server for offline analysis. Conventional echocardiographic parameters were measured according to the recommendations of the American Society of Echocardiography (ASE). ${ }^{25} 26$ The early diastolic mitral annular tissue velocity (e') was measured in the apical 4-chamber view with the sample positioned at the septal annulus. The combined assessment of transmitral early diastolic velocity (E) and e' was used to calculate E/e'. The severity of valvular heart disease was assessed in accordance with ASE guidelines. ${ }^{27} 28$

\section{Follow-up}

The primary outcome was 1-year unplanned all-cause readmission after discharge. Readmission data were analysed only for Kitaishikai Hospital as it is the only provider of intensive cardiology care in the region. HF-specific readmission was assessed using a similar definition of inclusion criteria. The secondary outcome was 1-year unplanned all-cause readmission or death after discharge. Two cardiologists (SI and MS) reviewed each medical record to confirm mortality or they communicated through regular mail or phone calls to patients, their relatives or general practitioners. Patients were censored at the time of each outcome or at the end of follow-up (maximum 1 year).

\section{Statistical analysis}

Data were missing from $3.7 \%$ of the records, except for B-type natriuretic peptide (12\%). Missing data were assumed to be random and were addressed using a multiple imputation procedure using the Markov Chain Monte Carlo method. Five imputed data sets were created, and the results were pooled according to Rubin's protocol. ${ }^{29}$

Data are expressed as the median (IQR). The significances in differences between groups were assessed using the Mann-Whitney $U$ test. For categorical variables, the $\chi^{2}$ test or Fisher's exact tests were used as appropriate. Survival analysis was conducted using the Cox proportional hazard analysis. The simple association of study variables with readmission was assessed by univariate analysis. For multiple regression analyses, we constructed models including age, sex, discharge to a nursing home and each risk score. Optimum model candidates were selected based on clinically relevant parameters. Harrell's C statistic was used to evaluate model performance. ${ }^{30}$ Receiver operating characteristic (ROC) curves and the probability of each model calculated from multiple logistic regression were used to assess the incremental benefit of LTCI compared with the clinical risk scores for predicting the primary outcome. Comparisons of areas under ROC curves (AUC) were performed using the method suggested by DeLong et al. ${ }^{31}$ Reclassification was evaluated to assess the incremental benefit of adding LTCI to clinical risk scores using categorical and continuous net reclassification improvement (NRI) methods and integrated diagnostic improvement. ${ }^{32} 33$ To calculate categorical NRI, patients were classified within the quartile boundaries of each clinical risk score. The interaction of LTCI-readmission association according to groups with and without preserved left ventricular ejection fraction (EF) was assessed by Cox proportional hazard models. Owing to significant interaction with LTCI ( $p$ interaction $=0.03$ ) in unadjusted models, we performed stratified analyses by $\mathrm{EF}$ ( $\mathrm{EF} \geq 50 \%$ vs $\mathrm{EF}<50 \%)$. In addition, since the components of each score may be underestimated or overestimated if we simply incorporate LTCI into each score, we also confirmed the independence of LTCI for predicting 1-year all-cause readmission in the models including LTCI and all components for each score using the Cox multiple regression analysis. Similarly, the incremental benefit of LTCI was confirmed by comparing the set of patient-level predicted probabilities of readmission for each model including LTCI and components for each score to that including the components for each score only using NRI and comparisons of AUC curves. However, these analyses were not performed in subgroups to avoid overfitting.

Statistical analysis was performed using the Standard Statistical Software Package (SPSS) software V.20.0 (SPSS, Chicago, Illinois, USA) and R software V.3.0.2, http://cran.r-project.org/, and $\mathrm{p}<0.05$ indicates statistical significance.

\section{RESULTS}

\section{Patient characteristics}

Table 1 summarises the patient baseline clinical, therapeutic, imaging and socioeconomic parameters. These 
Table 1 Patients' characteristics

\begin{tabular}{|c|c|}
\hline Variables & $\begin{array}{l}\text { Overall } \\
(n=452)\end{array}$ \\
\hline Age (years) & $81(74-85)$ \\
\hline Male sex, n (\%) & $240(53)$ \\
\hline Body weight $(\mathrm{kg})$ & $50(43-58)$ \\
\hline Body mass index $\left(\mathrm{kg} / \mathrm{m}^{2}\right)$ & $21.5(19.2-24.5)$ \\
\hline Systolic blood pressure (mm Hg) & $113(102-126)$ \\
\hline Diastolic blood pressure (mm Hg) & $61(56-70)$ \\
\hline Heart rate $(/ \min )$ & $66(60-76)$ \\
\hline NHYA functional class at discharge (I/II/III/IV), n (\%) & 0/377/74/1 (0/84/16/0) \\
\hline Length of hospital stay (days) & $21(14-35)$ \\
\hline Admissions in past year, $\mathrm{n}(\%)$ & $115(25)$ \\
\hline First admission due to heart failure & $296(66)$ \\
\hline \multicolumn{2}{|l|}{ Habitus } \\
\hline Smoking (no/past/current), n (\%) & $270 / 137 / 45(60 / 30 / 10)$ \\
\hline Alcohol abuse, $\mathrm{n}(\%)$ & $7(2)$ \\
\hline \multicolumn{2}{|l|}{ Aetiology } \\
\hline IHD/HTN/valve/rhythm/DCM/HCM/PH/unknown, n (\%) & $\begin{array}{l}\text { 110/14/92/67/44/14/4/107 (24/3/20/15/10/ } \\
3 / 1 / 24)\end{array}$ \\
\hline \multicolumn{2}{|l|}{ Comorbidities } \\
\hline Hypertension, n (\%) & $217(48)$ \\
\hline Diabetes, n (\%) & $124(27)$ \\
\hline Dyslipidaemia, n (\%) & $125(28)$ \\
\hline Atrial fibrillation, $\mathrm{n}(\%)$ & $161(36)$ \\
\hline Angina, $\mathrm{n}(\%)$ & $85(19)$ \\
\hline Myocardial infarction, n (\%) & $100(22)$ \\
\hline Sustained VT or VF or advanced AVB, $n(\%)$ & $57(13)$ \\
\hline Peripheral vascular disease, $\mathrm{n}(\%)$ & $25(6)$ \\
\hline Chronic lung disease or COPD, n (\%) & $21(5)$ \\
\hline Cerebrovascular disease or stroke, n (\%) & 84 (19) \\
\hline Cognitive impairment, n (\%) & $48(11)$ \\
\hline Depression, n (\%) & $11(3)$ \\
\hline Connective tissue disease, $\mathrm{n}(\%)$ & $1(0)$ \\
\hline Gastric ulcer, n (\%) & $4(1)$ \\
\hline Solid organ tumour, n (\%) & $58(13)$ \\
\hline Cirrhosis, n (\%) & $13(3)$ \\
\hline \multicolumn{2}{|l|}{ Past therapies } \\
\hline Previous CABG, n (\%) & $22(5)$ \\
\hline Previous other cardiac surgery, n (\%) & $29(6)$ \\
\hline Previous PTCA, n (\%) & $81(18)$ \\
\hline Device (N/PPM/CRT-D/ICD), n (\%) & $403 / 46 / 2 / 1(90 / 9 / 1 / 0)$ \\
\hline \multicolumn{2}{|l|}{ Serum markers } \\
\hline B-type natriuretic peptide (pg/mL) & $220(115-420)$ \\
\hline Haemoglobin $(g / L)$ & $11.7(10.2-13.0)$ \\
\hline Blood urea nitrogen (mg/dL) & $23(17-31)$ \\
\hline Creatinine $(\mathrm{mg} / \mathrm{dL})$ & $1.0(0.8-1.4)$ \\
\hline eGFR $\left(\mathrm{mL} / \mathrm{min} / 1.73 \mathrm{~m}^{2}\right)$ & $47(33-60)$ \\
\hline Sodium (mmol/L) & $139(137-140)$ \\
\hline Serum albumin (mg/L) & $3.5(3.2-3.8)$ \\
\hline \multicolumn{2}{|l|}{ Medications } \\
\hline$\beta$ blockers, n (\%) & $293(65)$ \\
\hline ACEi/ARB, n (\%) & $305(68)$ \\
\hline Diuretics (loop or thiazide), n (\%) & $413(91)$ \\
\hline Aldosterone antagonist therapy, $\mathrm{n}(\%)$ & $278(62)$ \\
\hline Antiarrythmic drugs except digoxin, $n$ (\%) & $72(16)$ \\
\hline Digoxin, $\mathrm{n}(\%)$ & $70(16)$ \\
\hline Statin, $n(\%)$ & $124(27)$ \\
\hline Non-steroidal anti-inflammatory drugs, n (\%) & $20(4)$ \\
\hline Respiratory interventions (N/CPAP/ASV/HOT), n (\%) & $414 / 7 / 12 / 19(91 / 2 / 3 / 4)$ \\
\hline
\end{tabular}




\begin{tabular}{|c|c|}
\hline Variables & $\begin{array}{l}\text { Overall } \\
(n=452)\end{array}$ \\
\hline \multicolumn{2}{|l|}{ Echocardiographic variables } \\
\hline LV mass index $\left(\mathrm{g} / \mathrm{m}^{2}\right)$ & $120(95-149)$ \\
\hline LV end-diastolic volume (mL) & $77(56-106)$ \\
\hline LV end-systolic volume (mL) & $36(23-62)$ \\
\hline LV ejection fraction (\%) & $51(41-62)$ \\
\hline$e^{\prime}(\mathrm{cm} / \mathrm{s})$ & $4.4(3.3-5.6)$ \\
\hline E/e' & $18.8(13.8-25.2)$ \\
\hline Moderate to severe aortic stenosis, n (\%) & $53(12)$ \\
\hline Moderate to severe mitral regurgitation, $\mathrm{n}(\%)$ & $157(35)$ \\
\hline Moderate to severe aortic regurgitation, $\mathrm{n}(\%)$ & $114(25)$ \\
\hline \multicolumn{2}{|l|}{ Clinical scores } \\
\hline Score 1: Yale CORE Score (\%) & $19(17-23)$ \\
\hline Score 2: Krumholz score & $1(0-2)$ \\
\hline Score 3: Charlson comorbidity index & $3(2-4)$ \\
\hline \multicolumn{2}{|l|}{ Socioeconomic variables } \\
\hline Marital history, n (\%) & $420(93)$ \\
\hline Living alone, $\mathrm{n}(\%)$ & $80(18)$ \\
\hline Low income, $\mathrm{n}(\%)^{*}$ & $42(9)$ \\
\hline Number of generations in a household $(1 / 2 / 3 / 4), n(\%) \dagger$ & $218 / 147 / 83 / 4(48 / 33 / 18 / 1)$ \\
\hline Number of housemates & $1(1-3)$ \\
\hline Number of male housemates & $1(0-1)$ \\
\hline Number of female housemates & $1(0-1)$ \\
\hline $\begin{array}{l}\text { Main caregiver (spouse/son/daughter/brother/sister/one's in-laws/relative/ } \\
\text { unknown), } \mathrm{n}(\%)\end{array}$ & $\begin{array}{l}123 / 63 / 84 / 7 / 10 / 74 / 44 / 47(27 / 14 / 19 / 2 / 2 / 16 / \\
10 / 10)\end{array}$ \\
\hline Discharge to nursing home, $\mathrm{n}(\%)$ & $28(6)$ \\
\hline Receiving long-term care insurance, $\mathrm{n}(\%)$ & $166(37)$ \\
\hline Category of long-term care insurance (N/requiring help/long-term care), n (\%) & $286 / 50 / 116(63 / 11 / 26)$ \\
\hline
\end{tabular}

${ }^{*}$ Patients who were certified as residents for tax exemption.

†Single generation includes living alone, living with a partner and living in a nursing home. Data are expressed as the median (IQR). $\mathrm{ARB}$, angiotensin receptor blocker; ASV, adaptive servoventilation; AVB, atrioventricular block; CABG, coronary artery bypass graft surgery; COPD, chronic obstructive pulmonary disease; CPAP; continuous positive airway pressure; CRT-D, cardiac resynchronisation therapydefibrillator; DCM, dilated cardiomyopathy; HCM, hypertrophic cardiomyopathy; HOT, home oxygen therapy; HTN, hypertension; ICD, implantable cardioverter-defibrillator; IHD, ischaemic heart disease; LA, left atrial; LV, left ventricular; LVH, left ventricular hypertrophy; NYHA, New York Heart Association; PH, pulmonary hypertension; PPM, permanent pacemaker; PTCA, percutaneous transluminal coronary angioplasty; VF, ventricular fibrillation; VT, ventricular tachycardia.

findings conformed to an expected heterogeneous population of patients with $\mathrm{HF}$.

\section{Events}

Follow-up data were available for all 452 patients, with 193 $(43 \%)$ primary outcomes, all-cause readmission within 1 year after discharge. When the patients with HF were divided into groups with preserved EF $(\geq 50 \%)$ or reduced $\mathrm{EF}(<50 \%)$, the latter comprised 213 patients and 100 events $(47 \%)$, while the former included 239 patients and 93 events $(39 \%)$. HF deteriorated among 99 of the 193 readmitted patients $(51 \%)$. The most common reason for readmission other than $\mathrm{HF}$ was lung disease (28 events, 94 readmissions other than HF, 30\%). Patients with $\mathrm{HF}$ with preserved EF (52 readmissions other than HF per 93 all-cause readmissions, 56\%) had higher rates of readmission other than for $\mathrm{HF}$ compared with patients with $\mathrm{HF}$ with reduced $\mathrm{EF}$ (42 readmissions other than for $\mathrm{HF}$ per 100 all-cause readmissions, 42\%, p=0.053). In addition, 24 deaths were observed as a first event after discharge. Accordingly, secondary outcome, 1-year allcause readmission or death, occurred in 217 patients $(48 \%)$.

\section{Factors associated with primary outcome}

Table 2 shows the univariate Cox regression analyses of the existing risk scores and the socioeconomic parameters that were associated with the primary outcome. The primary outcome was significantly associated with clinical risk score 1, score 2, discharge to a nursing home, receiving LTCI and the category of LTCI.

\section{Analysis of patients with and without LTCI}

Online supplementary table S1 compares the characteristics of patients with and without LTCI. Patients with LTCI were significantly older, and comprised more females compared with those without LTCI. The characteristics of patients with LTCI compared with those without were as follows: significantly leaner; hospitalised longer after the index admission; higher frequency of admissions during the past year; higher 
Table 2 Univariate Cox regression analyses of the association between primary outcome and the existing clinical risk scores and socioeconomic parameters

\begin{tabular}{|c|c|}
\hline Variables & HR (95\% Cl), p Value \\
\hline \multicolumn{2}{|l|}{ Clinical scores } \\
\hline $\begin{array}{l}\text { Score 1: Yale CORE Score } \\
\text { (per 1\%) }\end{array}$ & 1.07 (1.04 to 1.10$),<0.01$ \\
\hline $\begin{array}{l}\text { Score 2: Krumholz score } \\
\text { (per 1) }\end{array}$ & 1.31 (1.13 to 1.53$),<0.01$ \\
\hline $\begin{array}{l}\text { Score 3: Charlson } \\
\text { comorbidity index (per 1) }\end{array}$ & 1.06 (0.99 to 1.14$), 0.07$ \\
\hline \multicolumn{2}{|l|}{ Socioeconomic variables } \\
\hline Marital history & 0.96 (0.56 to 1.66$), 0.89$ \\
\hline Living alone & 1.10 (0.76 to 1.58$), 0.62$ \\
\hline Low income & 1.07 (0.77 to 1.47$), 0.70$ \\
\hline $\begin{array}{l}\text { Number of generations in a } \\
\text { household (per } 1 \\
\text { generation) }\end{array}$ & 0.91 (0.79 to 1.04$), 0.16$ \\
\hline $\begin{array}{l}\text { Number of housemates (per } \\
1 \text { person) }\end{array}$ & 0.95 (0.86 to 1.04$), 0.26$ \\
\hline $\begin{array}{l}\text { Number of male } \\
\text { housemates (per } 1 \text { person) }\end{array}$ & 0.96 (0.82 to 1.12 ), 0.62 \\
\hline $\begin{array}{l}\text { Number of female } \\
\text { housemates (per } 1 \text { person) }\end{array}$ & 0.90 (0.77 to 1.05 ), 0.19 \\
\hline Discharge to nursing home & 1.76 (1.07 to 2.90$), 0.03$ \\
\hline $\begin{array}{l}\text { Receiving long-term care } \\
\text { insurance }\end{array}$ & 1.56 (1.18 to 2.08$),<0.01$ \\
\hline $\begin{array}{l}\text { Category of long-term care } \\
\text { insurance (per } 1 \text { grade) }\end{array}$ & $1.30(1.11-1.52),<0.01$ \\
\hline
\end{tabular}

prevalences of cerebrovascular disease, cognitive impairment and depression; lower serum albumin; increased use of non-steroidal anti-inflammatory drugs; and longer nursing home stays after hospital discharge. The types of main caregivers differed between groups.

\section{Independent associations of LTCI with outcomes}

Multiple Cox regression analyses (table 3) revealed that possession of an LTCI certificate was independently associated with primary outcome after adjusting for age, sex, discharge to a nursing home and each clinical score. The effect size of receiving LTCI was similar in every model (HR, 1.59 to 1.63). Similarly, possession of an LTCI certificate was independently associated with the secondary outcome after the same adjustments (see online supplementary table S2).

\section{Incremental benefit of LTCI for predicting primary outcome compared with the clinical scores}

The discriminative ability of each risk score for predicting primary outcome was poor (AUCs, 0.55 to 0.60 ) (see online supplementary figure S1). Although the AUCs were not significantly improved by adding the LTCI certificate to each risk score, further significant reclassification improvements were observed (see online supplementary table S3). However, the adjusted model performances remained modest (AUCs, 0.59 to 0.63 ).

\section{Independence and incremental benefit of LTCI after adjustment for all components in each score}

The effect sizes of the components for each score may depend on the characteristics of the study cohort, and this may affect the predictive performance of each score. Therefore, we confirmed the independence of LTCI for predicting primary outcome by adjusting for all components in each score. Similar to the situation where the LTCI certificate was simply adjusted by each score, the LTCI certificate was independently associated with readmission even after adjustment for all components in each score (adjusted by score 1 components, HR: 1.42, 95\% CI 1.02 to $1.98, \mathrm{p}=0.03$; adjusted by score 2 components, HR: $1.45,95 \%$ CI 1.08 to $1.94, \mathrm{p}=0.02$; adjusted by score 3 components, HR: $1.49,95 \%$ CI 1.10 to $2.01, \mathrm{p}=0.01)$. In addition, the model based on LTCI and all components for each score demonstrated better predictive ability than the model based on components for each score only (see online supplementary table S4 and figure $\mathrm{S} 2$ ).

\section{Association of LTCI and primary outcome in subgroups}

To assess the influence of cardiac function between LTCI and the primary outcome, patients were divided into two

Table 3 Independent characteristics associated with primary outcome (multiple Cox regression)

\begin{tabular}{|c|c|c|c|}
\hline Variables & $\begin{array}{l}\text { Yale CORE model } \\
\text { (Model } \chi^{2}=35.7 \text {, } \\
\text { c-statistics }=0.61) \\
\text { HR }(95 \% \mathrm{Cl}), \mathrm{p} \text { Value }\end{array}$ & $\begin{array}{l}\text { Krumholz model } \\
\text { (Model } \chi^{2}=25.1 \text {, } \\
\text { c-statistics }=0.60) \\
\text { HR }(95 \% \mathrm{Cl}), \mathrm{p} \text { Value }\end{array}$ & $\begin{array}{l}\text { Charlson model } \\
\text { (Model } \chi^{2}=16.6 \text {, } \\
\text { c-statistics }=0.57) \\
\text { HR }(95 \% \mathrm{Cl}), \mathrm{p} \text { Value }\end{array}$ \\
\hline Age (per 1-year increase) & 1.00 (0.98 to 1.01$), 0.69$ & 1.00 (0.98 to 1.01$), 0.61$ & 0.99 (0.98 to 1.01$), 0.40$ \\
\hline Male sex & 1.29 (0.96 to 1.73$), 0.10$ & 1.23 (0.91 to 1.66$), 0.17$ & 1.23 (0.91 to 1.66$), 0.19$ \\
\hline $\begin{array}{l}\text { Score 1: Yale CORE score (per 1\% } \\
\text { increase) }\end{array}$ & 1.07 (1.04 to 1.10$),<0.01$ & & \\
\hline Score 2: Krumholz score (per $1 \%$ increase) & & 1.29 (1.10 to 1.51$),<0.01$ & \\
\hline $\begin{array}{l}\text { Score 3: Charlson comorbidity index (per } \\
1 \% \text { increase) }\end{array}$ & & & 1.05 (0.98 to 1.13$), 0.19$ \\
\hline Discharge to nursing home & 1.41 (0.84 to 2.38$), 0.20$ & 1.42 (0.84 to 2.39$), 0.19$ & 1.40 (0.82 to 2.38$), 0.22$ \\
\hline Receiving long-term care insurance & $1.62(1.17$ to 2.24$),<0.01$ & 1.59 (1.15 to 2.19$),<0.01$ & 1.63 (1.17 to 2.25$),<0.01$ \\
\hline
\end{tabular}


subgroups according to EF. LTCI was associated with the primary outcome, which was independent of each clinical risk score in the subgroup of patients with HF with preserved $\mathrm{EF}$ but not in the subgroup of $\mathrm{HF}$ with reduced EF (table 4). In addition, adding LTCI to all risk scores led to a significantly improved model performance and reclassification in the subgroup of patients with $\mathrm{HF}$ with preserved EF (table 5 and figure 1) but not in the subgroup with reduced EF (see online supplementary table S5 and figure S3).

Further, the effect sizes for LTCI appeared to be larger in the group with preserved EF than in the group with reduced EF (Yale CORE model, $p$ interaction $=0.04$, Krumholz model, $\mathrm{p}$ interaction $=0.10$, Charlson model, $\mathrm{p}$ interaction $=0.049$ ).

\section{DISCUSSION}

In this study, we demonstrated that the LTCI certificate provided incremental value over the conventional clinical risk scores for predicting 1-year all-cause readmission of patients with confirmed HF, particularly those with preserved LVEF.

\section{Contribution of including LTCI in the validated risk score for predicting readmission}

Several models or validated risk scores are available for predicting readmission of patients with $\mathrm{HF}^{3-7}$ They mainly comprise comprehensive clinical and administrative parameters because the causes of readmission are diverse. However, the accuracies of these models are insufficient. ${ }^{3-7}$ The discriminatory power of the clinical

Table 4 Independent characteristics associated with primary outcome (multiple Cox regression) in the subgroups HF with reduced EF and HF with preserved EF

HF reduced EF; N=213, Primary outcome=100 (47\%)

\begin{tabular}{|c|c|c|c|}
\hline Variables & $\begin{array}{l}\text { Yale CORE model } \\
\text { (Model } \chi^{2}=20.5 \text {, } \\
\text { c-statistics }=0.61) \\
\text { HR }(95 \% \mathrm{Cl}), \mathrm{p} \text { Value }\end{array}$ & $\begin{array}{l}\text { Krumholz model } \\
\text { (Model } \chi^{2}=19.9, \\
\text { c-statistics }=0.62) \\
\text { HR }(95 \% \mathrm{Cl}), \mathrm{p} \text { Value }\end{array}$ & $\begin{array}{l}\text { Charlson model } \\
\text { (Model } \chi^{2}=5.3 \text {, } \\
\text { C-statistics }=0.57 \text { ) } \\
\text { HR }(95 \% \mathrm{Cl}), \mathrm{p} \text { Value }\end{array}$ \\
\hline Age (per 1-year increase) & 1.00 (0.98 to 1.02$), 0.69$ & 1.00 (0.98 to 1.02$), 0.68$ & $\begin{array}{l}0.99(0.97 \text { to } 1.01) \\
0.57\end{array}$ \\
\hline Male sex & 1.16 (0.76 to 1.77$), 0.51$ & 1.09 (0.71 to 1.69$), 0.69$ & $\begin{array}{l}1.13(0.74 \text { to } 1.75) \\
0.57\end{array}$ \\
\hline Score 1: Yale CORE score (per $1 \%$ increase) & $\begin{array}{l}1.09(1.04 \text { to } 1.13) \\
<0.01\end{array}$ & & \\
\hline Score 2: Krumholz score (per 1\% increase) & & $\begin{array}{l}1.57(1.26 \text { to } 1.96) \\
<0.01\end{array}$ & \\
\hline $\begin{array}{l}\text { Score 3: Charlson comorbidity index (per 1\% } \\
\text { increase) }\end{array}$ & & & $\begin{array}{l}1.08(0.97 \text { to } 1.20) \\
0.16\end{array}$ \\
\hline Discharge to nursing home & 1.45 (0.67 to 3.15$), 0.35$ & 1.65 (0.77 to 3.54$), 0.20$ & $\begin{array}{l}1.51(0.69 \text { to } 3.32) \\
0.30\end{array}$ \\
\hline Receiving long-term care insurance & 1.30 (0.81 to 2.08$), 0.28$ & 1.24 (0.77 to 1.98$), 0.37$ & $\begin{array}{l}1.22(0.76 \text { to } 1.96) \\
0.41\end{array}$ \\
\hline
\end{tabular}

HF preserved EF; N=239, Primary outcome=93 (39\%)

\begin{tabular}{|c|c|c|c|}
\hline Variables & $\begin{array}{l}\text { Yale CORE model } \\
\text { (Model } \chi^{2}=18.6 \text {, } \\
\text { c-statistics }=0.61) \\
\text { HR }(95 \% \mathrm{Cl}), \mathrm{p} \text { Value }\end{array}$ & $\begin{array}{l}\text { Krumholz model } \\
\text { (Model } \chi^{2}=14.7, \\
\text { c-statistics }=0.60) \\
\text { HR }(95 \% \mathrm{Cl}), p \text { Value }\end{array}$ & $\begin{array}{l}\text { Charlson model } \\
\text { (Model } \chi^{2}=14.5 \text {, } \\
\text { c-statistics }=0.59 \text { ) } \\
\text { HR }(95 \% \mathrm{Cl}), \mathrm{p} \text { Value }\end{array}$ \\
\hline Age (per 1-year increase) & $\begin{array}{l}1.01 \text { (0.97 to } 1.04) \\
0.77\end{array}$ & $\begin{array}{l}1.00(0.97 \text { to } 1.03) \\
0.84\end{array}$ & 1.00 (0.97 to 1.03$), 0.97$ \\
\hline Male sex & $\begin{array}{l}1.33(0.87 \text { to } 2.03) \\
0.18\end{array}$ & $\begin{array}{l}1.27(0.83 \text { to } 1.94) \\
0.26\end{array}$ & 1.25 (0.81 to 1.92$), 0.31$ \\
\hline Score 2: Krumholz score (per $1 \%$ increase) & & $\begin{array}{l}1.09 \text { (0.87 to } 1.37) \text {, } \\
0.45\end{array}$ & \\
\hline $\begin{array}{l}\text { Score 3: Charlson comorbidity index (per 1\% } \\
\text { increase) }\end{array}$ & & & 1.03 (0.93 to 1.14$), 0.54$ \\
\hline Discharge to nursing home & 1.33 (0.65 to 2.72$), 0.44$ & $1.31(0.64$ to 2.70$), 0.46$ & $1.29(0.62$ to 2.69$), 0.49$ \\
\hline
\end{tabular}


Table 5 Change in risk classification for the probability of primary outcome using a model including long-term care insurance compared with a model using each score only in the subgroup heart failure with preserved ejection fraction

\begin{tabular}{|c|c|c|c|c|c|c|c|c|}
\hline & & \multicolumn{4}{|l|}{ Score 1+LTCI } & \multicolumn{2}{|l|}{ Reclassified } & \multirow{2}{*}{$\begin{array}{l}\text { Net correctly } \\
\text { reclassified, \%* }\end{array}$} \\
\hline & & Quartile $1(<33 \%)$ & Quartile 2 (34-37\%) & Quartile 3 (38-41\%) & Quartile $4(<42 \%)$ & Increased risk & Decreased risk & \\
\hline \multicolumn{9}{|c|}{ Readmission (93) } \\
\hline \multirow{4}{*}{ Score 1} & Quartile 1 (<33\%) & 13 & 7 & 3 & 0 & \multirow[t]{4}{*}{27} & \multirow[t]{4}{*}{23} & \multirow[t]{4}{*}{4.3} \\
\hline & Quartile 2 (34-37\%) & 9 & 0 & 2 & 4 & & & \\
\hline & Quartile 3 (38-41\%) & 9 & 2 & 0 & 11 & & & \\
\hline & Quartile 4 (<42\%) & 0 & 1 & 2 & 30 & & & \\
\hline \multicolumn{9}{|c|}{ No readmission (146) } \\
\hline \multirow[t]{4}{*}{ Score 1} & Quartile 1 (<33\%) & 27 & 2 & 5 & 0 & \multirow[t]{4}{*}{33} & \multirow[t]{4}{*}{67} & \multirow[t]{4}{*}{23.3} \\
\hline & Quartile 2 (34-37\%) & 29 & 0 & 8 & 7 & & & \\
\hline & Quartile 3 (38-41\%) & 14 & 12 & 0 & 11 & & & \\
\hline & Quartile $4(<42 \%)$ & 0 & 1 & 11 & 19 & & & \\
\hline \multirow{5}{*}{\multicolumn{2}{|c|}{$\begin{array}{l}\text { Categorical NRI† } \\
\text { Continuous NRI } \\
\text { IDI } \\
\end{array}$}} & & & & & & & \multirow{5}{*}{$\begin{array}{l}0.28 \S \\
0.55 \S \\
0.04 \S \\
\text { Net correctly } \\
\text { reclassified, \%* }\end{array}$} \\
\hline & & & & & & & & \\
\hline & & & & & & & & \\
\hline & & \multicolumn{4}{|l|}{ Score 2+LTCI } & \multicolumn{2}{|l|}{ Reclassified } & \\
\hline & & Quartile 1 (<34\%) & Quartile 2 (35-38\%) & Quartile 3 (39-42\%) & Quartile $4(<43 \%)$ & Increased risk & Decreased risk & \\
\hline \multicolumn{9}{|c|}{ Readmission (93) } \\
\hline \multirow[t]{4}{*}{ Score 2} & Quartile 1 (<34\%) & 7 & 0 & 0 & 0 & \multirow[t]{4}{*}{19} & \multirow[t]{4}{*}{42} & \multirow[t]{4}{*}{-24.7} \\
\hline & Quartile 2 (35-38\%) & 17 & 14 & 0 & 0 & & & \\
\hline & Quartile 3 (39-42\%) & 16 & 0 & 1 & 19 & & & \\
\hline & Quartile $4(<43 \%)$ & 0 & 0 & 9 & 10 & & & \\
\hline \multicolumn{9}{|c|}{ No readmission (146) } \\
\hline \multirow[t]{4}{*}{ Score 2} & Quartile $1(<34 \%)$ & 6 & 0 & 0 & 0 & \multirow[t]{4}{*}{19} & \multirow[t]{4}{*}{97} & 53.4 \\
\hline & Quartile 2 (35-38\%) & 42 & 15 & 0 & 0 & & & \\
\hline & Quartile 3 (39-42\%) & 35 & 0 & 1 & 19 & & & \\
\hline & Quartile $4(<43 \%)$ & 0 & 0 & 20 & 8 & & & \\
\hline Categoric & cal NRI† & & & & & & & $0.29 \S$ \\
\hline Continuo & us NRI & & & & & & & $0.43 \S$ \\
\hline IDI & & & & & & & & $0.03 \S$ \\
\hline & & Score $3+\mathrm{LTCl}$ & & & & Reclassified & & Net correctly \\
\hline & & Quartile 1 (<35\%) & Quartile 2 (36-37\%) & Quartile 3 (38-40\%) & Quartile $4(<41 \%)$ & Increased risk & Decreased risk & reclassified, \%* \\
\hline Readmis & sion (93) & & & & & & & \\
\hline Score 3 & Quartile $1(<35 \%)$ & 11 & 0 & 0 & 5 & 21 & 32 & -11.8 \\
\hline & Quartile 2 (36-37\%) & 7 & 0 & 0 & 9 & & & \\
\hline & Quartile 3 (38-40\%) & 8 & 0 & 0 & 7 & & & \\
\hline & Quartile $4(<41 \%)$ & 13 & 0 & 4 & 29 & & & \\
\hline
\end{tabular}




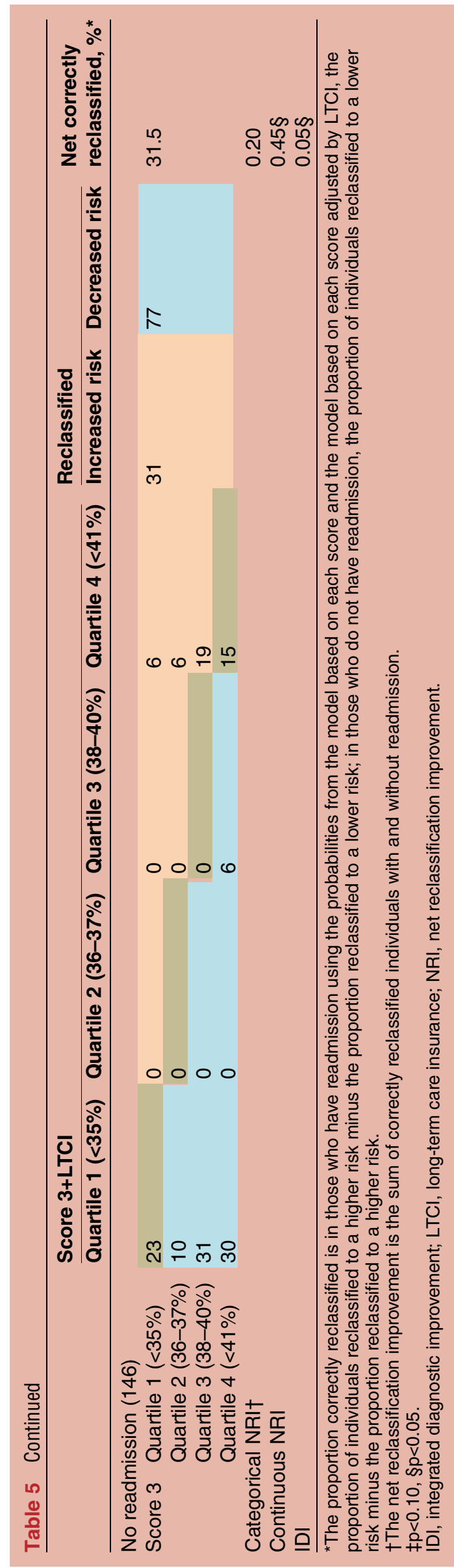

scores measured in this study was comparable with that of previous studies. ${ }^{3-7}$ These findings suggest that important predictors of readmission are not captured in contemporary data sets or are not incorporated in the existing models. ${ }^{3-7}$

Ageing patients with $\mathrm{HF}$ are increasing common in an ultra-ageing society. ${ }^{34}$ The majority of elderly patients with $\mathrm{HF}$ are frail and suffer from multiple chronic comorbidities such as chronic lung disease, renal failure and cerebrovascular disease. ${ }^{3}{ }^{35}$ Frailty plays an important role in predicting adverse outcomes, because it may lead to $\mathrm{HF}$ as well as other comorbidities requiring hospitalisation. ${ }^{15-19}{ }^{36}$ However, accurate quantification is challenging because frailty is associated with the complicated process of ageing, and there is no standard definition of frailty, which most likely accounts for its omission from existing clinical scores.

The recipients of LTCI are demonstrably frail and have mental and physical impairments requiring sufficient social support. Moreover, in this study, the patients with LCTI were likely to be more frail compared with those without LTCI. These considerations suggest that the LTCI certificate may reflect frailty and consequently has an incremental benefit compared with conventional clinical risk scores for predicting readmission of patients with HF. Interestingly, Medicare coverage of citizens in the USA aged $\geq 65$ years is a risk factor for readmission of patients with $\mathrm{HF}^{7}$ and may be explained by the mechanism revealed here.

However, the use of the LTCI system should normally prevent adverse outcomes of patients with HF because of the social care provided. The independent positive association of the LTCI certificate with readmission determined in this study suggests that the LTCI certificate, the surrogate of frailty, is a stronger risk factor compared with the benefits provided by LTCI.

Interestingly, adding LTCI certificate possession to the models appeared to correctly reclassify patients thought to have moderate risk on conventional grounds into a lower risk group. This suggests that the LTCI certificate status may be useful for correcting pseudopositive estimation based on conventional clinical score.

For the clinician, confirming the LTCI certificate may be easier to estimate frailty rather than measuring existing frailty scores in the real clinical setting. From this aspect, the LTCI certificate may contribute to a simple prediction of readmission for HF.

However, the model performance was still inadequate after adjustment using only the LTCI certificate as a proxy for frailty. Risk prediction of readmission in HF is complex, so there is a need for further understanding of the factors that dominantly influence readmission risk. In addition, the present retrospective study focused on patients at a single centre; multicentre studies with larger diverse cohorts must be conducted to confirm these results. 
Score 1:

Yale CORE score

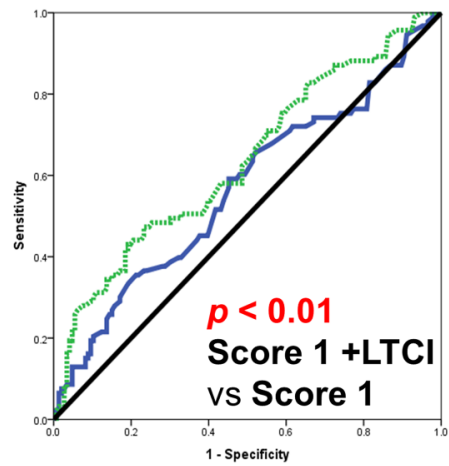

Sources of the curves

II Score 1+ LTCI
(AUC=0.64, 95\%Cl: 0.56-0.71)
Score 1
(AUC $=0.57,95 \% \mathrm{Cl}: 0.49-0.64)$
Score 2:

Krumholz score

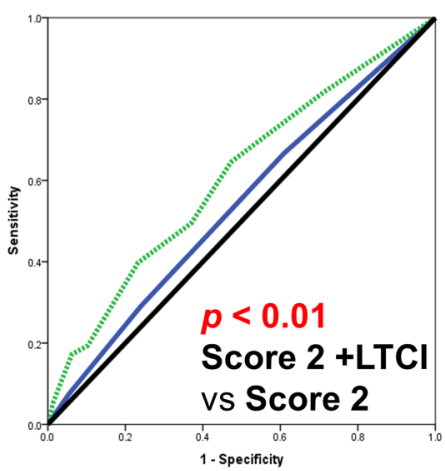

Sources of the curves

III Score 2 + LTCI

(AUC=0.61, 95\%Cl: 0.53-0.68)

- Score 2

(AUC=0.54, 95\%Cl: 0.46-0.61)
Score 3:

Charlson comorbidity index

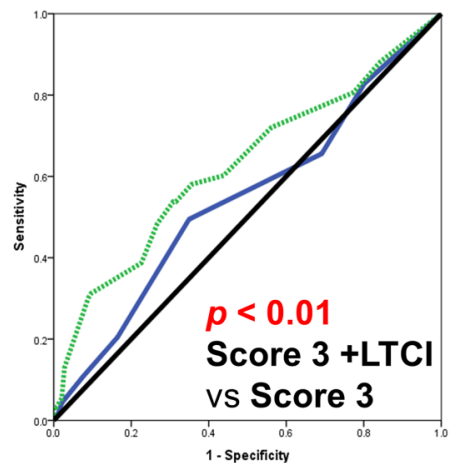

Sources of the curves

III Score 3 + LTCI

(AUC=0.63, 95\% $\mathrm{Cl}: 0.55-0.70$ )

$=$ Score 3

(AUC=0.54, 95\%Cl: 0.47-0.62)

Figure 1 Receiver operating characteristic curves for the association of primary outcome using each clinical risk score and each clinical risk score adjusted for long-term care insurance certificate possession in the subgroup of patients with heart failure with preserved ejection fraction. AUC, area under a receiver operating characteristic curve; LTCI, long-term care insurance.

\section{Socioeconomic parameters other than LTCI}

Although some studies have shown that socioeconomic factors are important mediators of the risk of readmission for patients with $\mathrm{HF}^{7} 81037$ most factors did not contribute to the prediction of readmission in this study. A recent systematic review found an inconsistent association between income and readmission of patients with HF. ${ }^{10}$ Japan provides medical insurance for the entire nation, as well as a high-cost medical expense benefit called the 'ceiling-medical amount' system. Thanks to these systems, Japan can provide medical care irrespective of its citizens' incomes. Therefore, this might account for the absence of an association between low income and readmission in the present cohort.

Living alone and marital status are risk factors for readmission in patients with $\mathrm{HF}^{3}{ }^{10}$ Ozu City is located in a rural area, and the proportion of multigenerational family structures, which is the traditional pattern of Japanese households. ${ }^{38}$ These factors might explain the low rate of single or unmarried patients and their lack of association with readmission in this study.

Although discharge to nursing home decreases readmissions in the USA, ${ }^{3} 7^{10}$ it was positively associated with readmission in this study and therefore LTCI might account for this inconsistency. The recipients of LTCI frequently use nursing homes, and they might be at greater risk for readmission after their discharge compared with patients discharged under normal circumstances.

A recent study found that self-reported socioeconomic variables were not dominant factors for predicting readmission risk of patients with $\mathrm{HF}^{9}$ The objective social parameter LTCI, including frailty, may contribute to the prediction of readmission.

\section{Stronger association of LTCI and readmission of patients with $\mathrm{HF}$ with preserved $\mathrm{EF}$}

Approximately $50 \%$ of readmissions of patients with $\mathrm{HF}$ is related to HF, not worsening, ${ }^{2}$ which is consistent with the present findings. Further, patients with HF with preserved EF had a higher rate of readmission compared with those categorised as other than HF, as well as those with HF with reduced EF. These findings may be explained by the association of increased age and a higher incidence of comorbidities of patients with HF with preserved $\mathrm{EF}$ compared with patients with $\mathrm{HF}$ with reduced $\mathrm{EF}^{39}$ which suggests that patients with $\mathrm{HF}$ with preserved EF may be more frail compared with patients with HF with reduced EF. Furthermore, in this study, patients with HF with preserved EF were significantly older compared to those with reduced EF (82 (IQR 7685 ) vs 79 (IQR 72-85) years, $\mathrm{p}<0.01$ ). Therefore, the backgrounds of patients with HF with preserved EF may be associated with those of the recipients of LTCI and may therefore lead to a stronger association between LTCI and readmission of patients with HF with preserved EF.

\section{Study limitations}

Our data should be interpreted according to the study's limitations. First, the original outcomes according to conventional clinical scores and index codes did not perfectly match outcomes in this study. A clinical risk score for predicting intermediate-term readmission for HF validated in a large Japanese HF patient cohort would be 
ideal, but no such metric is yet available. In addition, although several predictive models for readmission due to HF have been reported, it is unknown which ones are most applicable in the clinical setting. ${ }^{3}$ Therefore, we have applied three such scores. Second, the HRs for each risk score were expressed per unit increase in each score, which could be misleading as the possible range of values for each score in the study population greatly differs. Third, this retrospective analysis may have been biased by unmeasured confounders, although we conducted a comprehensive analysis of conventional clinical risks. Additionally, several important socioeconomic parameters, such as education, were not available in patients' records. Fourth, since some socioeconomic parameters including LTCI are not necessarily universal and depend on the insurance system in each country, this location bias may not be generalised to other health systems. However, like recognition of Medicare as an HF risk in the USA, ${ }^{7}$ the present results will be useful for several countries applying the LTCI system. Fifth, frailty, which might serve as a major contributor of the LTCI certificate, was not quantified. However, accurate estimation of frailty is quite difficult. Moreover, the LTCI certificate might more accurately reflect frailty compared with existing frailty scores because it can only be comprehensively assessed through the efforts of experts in diverse disciplines. Further, the characteristics of patients who receive LTCI might help estimate their degrees of frailty. In addition, several studies have shown that patients with LTCI are indeed frail. ${ }^{14} 4041$ Sixth, we analysed only three risk scores. Therefore, the incremental value of LTCI over other risk scores was unknown. However, most predictive models were not available as predictive scores and some of the variables included in other risk scores could not be captured retrospectively. ${ }^{4} 7{ }^{24}$ Seventh, although most regular patients would most likely be admitted to Kitaishikai Hospital, some patients may have been admitted to other hospitals. However, this possibility is most likely small considering Ozu City's rural location and the status of Kitaishikai Hospital as a core treatment centre for cardiovascular disorders. Finally, data for the frequency and efficacy of using social services under LTCI were not available for each patient. The effects of social services might confound the association of the LTCI certificate with readmission.

\section{Conclusions}

Patients having an LTCI certificate appeared to be frail. Possession of an LTCI certificate was independently associated with 1-year all-cause readmission even after adjustment of all validated clinical risk scores in elderly patients with HF. LTCI certificate possession as a proxy for frailty significantly improved the performance of readmission risk models, especially in patients with $\mathrm{HF}$ with preserved EF, but the model performance remained modest. Nonetheless, incorporating the component of frailty into conventional clinical risk models may be important for better prediction of readmission risk.
Acknowledgements The authors gratefully acknowledge the assistance of Masaru Morino in compiling the outcome data.

Contributors KT extracted, cleaned and analysed the data, conducted the statistical analysis, and drafted and revised the paper. MS designed the protocol and extracted the data and revised the draft paper. SI extracted the data and revised the draft paper. TM and HA extracted the data. TS, AO, SI and $\mathrm{JH}$ contributed to conception of the study and revised the manuscript. All authors had full access to the data and are guarantors for the study.

\section{Competing interests None declared.}

Ethics approval The study was approved by the ethics committee of Ehime University Graduate School of Medicine (number 1512001).

Provenance and peer review Not commissioned; externally peer reviewed.

Data sharing statement No additional data are available.

Open Access This is an Open Access article distributed in accordance with the Creative Commons Attribution Non Commercial (CC BY-NC 4.0) license, which permits others to distribute, remix, adapt, build upon this work noncommercially, and license their derivative works on different terms, provided the original work is properly cited and the use is non-commercial. See: http:// creativecommons.org/licenses/by-nc/4.0/

\section{REFERENCES}

1. Gheorghiade M, Vaduganathan M, Fonarow GC, et al. Rehospitalization for heart failure: problems and perspectives. J Am Coll Cardiol 2013;61:391-403.

2. O'Connor CM, Miller AB, Blair JE, et al. Causes of death and rehospitalization in patients hospitalized with worsening heart failure and reduced left ventricular ejection fraction: results from Efficacy of Vasopressin Antagonism in Heart Failure Outcome Study with Tolvaptan (EVEREST) program. Am Heart J 2010;159:841-9.e1.

3. Saito M, Negishi K, Marwick TH. Meta-analysis of risks for short-term readmission in patients with heart failure. Am J Cardiol 2016;117:626-32.

4. Rahimi K, Bennett D, Conrad N, et al. Risk prediction in patients with heart failure: a systematic review and analysis. JACC Heart failure 2014;2:440-6.

5. Keenan PS, Normand SL, Lin Z, et al. An administrative claims measure suitable for profiling hospital performance on the basis of 30-day all-cause readmission rates among patients with heart failure. Circ Cardiovasc Qual Outcomes 2008;1:29-37.

6. Krumholz HM, Chen YT, Wang Y, et al Predictors of readmission among elderly survivors of admission with heart failure. Am Heart $J$ 2000;139:72-7.

7. Philbin EF, DiSalvo TG. Prediction of hospital readmission for heart failure: development of a simple risk score based on administrative data. J Am Coll Cardiol 1999;33:1560-6.

8. Hersh AM, Masoudi FA, Allen LA. Postdischarge environment following heart failure hospitalization: expanding the view of hospital readmission. J Am Heart Assoc 2013;2:e000116.

9. Krumholz HM, Chaudhry SI, Spertus JA, et al. Do non-clinical factors improve prediction of readmission risk?: results from the Tele-Hf study. JACC Heart failure 2016;4:12-20.

10. Damiani G, Salvatori E, Silvestrini G, et al. Influence of socioeconomic factors on hospital readmissions for heart failure and acute myocardial infarction in patients 65 years and older: evidence from a systematic review. Clin Interv Aging 2015;10:237-45.

11. Matsuda S, Yamamoto M. Long-term care insurance and integrated care for the aged in Japan. Int J Integr Care 2001;1:e28.

12. Bakx $P$, de Meijer $C$, Schut $F$, et al. Going formal or informal, who cares? The influence of public long-term care insurance. Health Econ 2015;24:631-43.

13. Rhee JC, Done N, Anderson GF. Considering long-term care insurance for middle-income countries: comparing South Korea with Japan and Germany. Health Policy 2015;119:1319-29.

14. Akune $\mathrm{T}$, Muraki $\mathrm{S}$, Oka $\mathrm{H}$, et al. Incidence of certified need of care in the long-term care insurance system and its risk factors in the elderly of Japanese population-based cohorts: the ROAD study. Geriatr Gerontol Int 2014;14:695-701.

15. Vidan MT, Blaya-Novakova V, Sanchez E, et al. Prevalence and prognostic impact of frailty and its components in non-dependent elderly patients with heart failure. Eur J Heart Fail 2016;18:869-75.

16. Sanchez E, Vidan MT, Serra JA, et al. Prevalence of geriatric syndromes and impact on clinical and functional outcomes in older patients with acute cardiac diseases. Heart 2011;97:1602-6. 
17. Gastelurrutia $\mathrm{P}$, Lupon J, Altimir S, et al. Effect of fragility on quality of life in patients with heart failure. Am J Cardiol 2013;112:1785-9.

18. Khan H, Kalogeropoulos AP, Georgiopoulou VV, et al. Frailty and risk for heart failure in older adults: the health, ageing, and body composition study. Am Heart J 2013;166:887-94.

19. McNallan SM, Singh M, Chamberlain AM, et al. Frailty and healthcare utilization among patients with heart failure in the community. JACC Heart Fail 2013;1:135-41.

20. Rimland JM, Abraha I, Luchetta ML, et al. Validation of chronic obstructive pulmonary disease (COPD) diagnoses in healthcare databases: a systematic review protocol. BMJ Open 2016;6:e011777.

21. McMurray JJ, Adamopoulos S, Anker SD, et al. ESC guidelines for the diagnosis and treatment of acute and chronic heart failure 2012: the Task Force for the Diagnosis and Treatment of Acute and Chronic Heart Failure 2012 of the European Society of Cardiology. Developed in collaboration with the Heart Failure Association (HFA) of the ESC. Eur J Heart Fail 2012;14:803-69.

22. Charlson ME, Pompei $\mathrm{P}$, Ales $\mathrm{KL}$, et al. A new method of classifying prognostic comorbidity in longitudinal studies: development and validation. J Chronic Dis 1987;40:373-83.

23. Giamouzis G, Kalogeropoulos A, Georgiopoulou V, et al. Hospitalization epidemic in patients with heart failure: risk factors, risk prediction, knowledge gaps, and future directions. J Card Fail 2011;17:54-75.

24. Ross JS, Mulvey GK, Stauffer B, et al. Statistical models and patien predictors of readmission for heart failure: a systematic review. Arch Intern Med 2008;168:1371-86.

25. Lang RM, Bierig M, Devereux RB, et al. Recommendations for chamber quantification: a report from the American Society of Echocardiography's Guidelines and Standards Committee and the Chamber Quantification Writing Group, developed in conjunction with the European Association of Echocardiography, a branch of the European Society of Cardiology. J Am Soc Echocardiogr 2005;18:1440-63.

26. Nagueh SF, Appleton CP, Gillebert TC, et al. Recommendations for the evaluation of left ventricular diastolic function by echocardiography. Eur J Echocardiogr 2009;10:165-93.

27. Zoghbi WA, Enriquez-Sarano M, Foster E, et al. Recommendations for evaluation of the severity of native valvular regurgitation with two-dimensional and Doppler echocardiography. J Am Soc Echocardiogr 2003;16:777-802.

28. Baumgartner $\mathrm{H}$, Hung J, Bermejo J, et al. Echocardiographic assessment of valve stenosis: EAE/ASE recommendations for clinical practice. J Am Soc Echocardiogr 2009;22:1-23; quiz 101-2.
29. Little RJ, Rubin DB. Statistical analysis with missing data. New York, NY: John Wiley, 1987:150.

30. Harrell FE Jr, Lee KL, Mark DB. Multivariable prognostic models: issues in developing models, evaluating assumptions and adequacy, and measuring and reducing errors. Stat Med 1996;15:361-87.

31. DeLong ER, DeLong DM, Clarke-Pearson DL. Comparing the areas under two or more correlated receiver operating characteristic curves: a nonparametric approach. Biometrics 1988;44:837-45.

32. Pencina MJ, D'Agostino RB Sr, D'Agostino RB Jr, et al. Evaluating the added predictive ability of a new marker: from area under the ROC curve to reclassification and beyond. Stat Med 2008;27:157-72; discussion 207-12.

33. Mielniczuk LM, Tsang SW, Desai AS, et al. The association between high-dose diuretics and clinical stability in ambulatory chronic heart failure patients. J Card Fail 2008;14:388-93.

34. Okura Y, Ramadan MM, Ohno Y, et al. Impending epidemic: future projection of heart failure in Japan to the year 2055. Circ $J$ 2008;72:489-91.

35. Murad K, Kitzman DW. Frailty and multiple comorbidities in the elderly patient with heart failure: implications for management. Heart Fail Rev 2012;17:581-8.

36. Kojima G. Frailty as a predictor of hospitalisation among community-dwelling older people: a systematic review and meta-analysis. J Epidemiol Community Health 2016;70:722-9.

37. Amarasingham R, Moore BJ, Tabak YP, et al. An automated model to identify heart failure patients at risk for 30-day readmission or death using electronic medical record data. Med Care 2010;48:981-8.

38. Statistic Bureau, Ministry of Internal Affairs and Communications (Japan). Summary of the Results of Population Census of Japan. 2010. http://www.stat.go.jp/english/data/kokusei/2010/final_en/pdf/ summary.pdf (accessed 31 May 2016).

39. Owan TE, Hodge DO, Herges RM, et al. Trends in prevalence and outcome of heart failure with preserved ejection fraction. $N$ Engl J Med 2006;355:251-9.

40. Mitoku K, Masaki N, Ogata Y, et al. Vision and hearing impairments, cognitive impairment and mortality among long-term care recipients: a population-based cohort study. BMC Geriatr 2016;16:112.

41. Fukutomi E, Okumiya K, Wada T, et al. Relationships between each category of 25 -item frailty risk assessment (Kihon Checklist) and newly certified older adults under Long-Term Care Insurance: a 24-month follow-up study in a rural community in Japan. Geriatr Gerontol Int 2015;15:864-71. 